\title{
Observer design for switching nonlinear systems
}

\author{
Zsófia Lendek*, Paula Raica*, Jimmy Lauber ${ }^{\dagger}$, and Thierry Marie Guerra ${ }^{\dagger}$ \\ * Department of Automation, Technical University of Cluj-Napoca, \\ Memorandumului 28, 400114 Cluj-Napoca, Romania. \\ \{zsofia.lendek, paula.raica\}@aut.utcluj.ro \\ ${ }^{\dagger}$ University of Valenciennes and Hainaut-Cambresis, \\ LAMIH UMR CNRS 8201, Le Mont Houy, \\ 59313 Valenciennes Cedex 9, France. \\ \{jlauber, guerra\}@univ-valenciennes.fr
}

\begin{abstract}
In this paper we consider observer design for discrete-time switching systems represented by Takagi-Sugeno fuzzy models. For the design we use a switching Lyapunov function defined on the switches. In order to develop stability conditions for the estimation error dynamics, we consider the variation of this function along possible switches. The conservativeness of the approach is reduced by considering the $\alpha$ sample variation of the Lyapunov function. This approach can bring solutions to observer design for some switching systems with unobservable and unstable local models. The developed conditions are illustrated on a numerical example.

Index Terms-switching systems, Takagi-Sugeno fuzzy models, observer design, periodic systems, nonquadratic Lyapunov function
\end{abstract}

\section{INTRODUCTION}

Switching systems are usually described by continuous and discrete dynamics, and their interactions. For such systems, one can analyze the stability [1] of the whole system assuming that the switching sequence is known or not known and design switching observers and controllers. In this paper we consider observer design for switching nonlinear systems represented by Takagi-Sugeno (TS) fuzzy models.

Takagi-Sugeno (TS) fuzzy systems [2] are convex combinations of local linear models. For the analysis and synthesis of TS systems the direct Lyapunov approach has been used. The design conditions are derived in the form of linear matrix inequalities (LMIs), which can be solved using available algorithms. To derive the conditions, next to classical quadratic Lyapunov functions [3]-[5], recently nonquadratic Lyapunov functions [6]-[8] have been used, which have shown a real improvement in the design conditions [6], [9]-[11].

Switching TS systems have been investigated in the last decades mainly in the continuous case where the stability is based on the use of a quadratic Lyapunov function [12]-[15] or a piecewise one [16], [17]. For discrete-time TS models, the number of results is limited [18], [19].

Most of the existing results on switching systems concern linear subsystems, such as the one in [20], where stabilization in the presence of input saturation and uncertainties is considered, or [21] which considers the computation of the modedependent dwell-time. Observability for switching discretetime linear systems has been investigated in [22].
In this paper, we derive relaxed conditions for the observer design of switching TS systems. For this, we use a switching nonquadratic Lyapunov function and make use of its variation over possible switches. We assume that although the exact switching sequence is not known, the set of all the admissible switches is known. Furthermore, once a subsystem is activated, it will remain active for a number of samples, for which minimum and maximum bounds are known.

The structure of the paper is as follows. Section II presents the notations used in this paper. The development of observer design conditions is presented in Section III. Section IV discusses the developed conditions and illustrates their use on a numerical example. Section V concludes the paper.

\section{Preliminaries}

In this paper we consider observer design for discrete-time switching TS systems. We consider subsystems of the form

$$
\begin{aligned}
\boldsymbol{x}_{k+1} & =\sum_{i=1}^{r} h_{i}\left(\boldsymbol{z}_{k}\right)\left(A_{j, i} \boldsymbol{x}_{k}+B_{j, i} \boldsymbol{u}_{k}\right) \\
\boldsymbol{y}_{k} & =\sum_{i=1}^{r} h_{i}\left(\boldsymbol{z}_{k}\right) C_{j, i} \boldsymbol{x}_{k}
\end{aligned}
$$

or, in a shorthand notation

$$
\begin{aligned}
\boldsymbol{x}_{k+1} & =A_{j, z} \boldsymbol{x}_{k}+B_{j, z} \boldsymbol{u}_{k} \\
\boldsymbol{y}_{k} & =C_{j, z} \boldsymbol{x}_{k}
\end{aligned}
$$

where $j$ is the number of the subsystem, $j=1,2, \ldots, n_{\mathrm{s}}, n_{\mathrm{s}}$ being the number of the subsystems, $\boldsymbol{x}$ denotes the state vector, $r$ is the number of rules, $z$ denotes the scheduling vector, $h_{i}$, $i=1,2, \ldots, r$ are normalized membership functions, and $A_{j, i}, B_{j, i}, C_{j, i}, i=1,2, \ldots, r, j=1,2, \ldots, n_{\mathrm{s}}$, are the local models. The scheduling vector is assumed to be available online, but not in advance.

For the easier notation, we use a directed graph representation of the switching system (1). The graph associated to (1) is $\mathcal{G}=\{\mathcal{V}, \mathcal{E}\}$, where $\mathcal{V}$ denotes the set of vertices or subsystems and $\mathcal{E}$ denotes the set of admissible switches. As such, $\left(v_{i}, v_{j}\right) \in \mathcal{E}$ if a switch from subsystem $i$ to subsystem $j$ is possible. Note that we assume that self-transitions are also possible: these correspond to the subsystem being active for more than one sample. 
A path $\mathcal{P}\left(v_{i}, v_{j}\right)$ between two vertices $v_{i}$ and $v_{j}$ in the graph $\mathcal{G}$ is a sequence of vertices $\mathcal{P}\left(v_{i}, v_{j}\right)=\left[v_{p_{1}}, v_{p_{2}}, \ldots, v_{p_{n_{p}}}\right]$ so that $v_{i}=v_{p_{1}}, v_{j}=v_{p_{n_{p}}}$, and $\left(v_{p_{k}}, v_{p_{k+1}}\right) \in \mathcal{E}, p_{k}=$ $1,2, \ldots, n_{p}-1$. A path in a graph associated to a switching system corresponds to a switching law. The length of a path is given by the number of edges it contains.

The notations above are illustrated on the following example.

Example 1. Consider a switching system composed of 4 subsystem:

$$
\boldsymbol{x}_{k+1}=A_{i, z} \boldsymbol{x}_{k}
$$

for $i=1,2,3,4$, and with admissible switches $(2,1),(2,3)$, $(3,1),(4,2),(1,4)$. Each subsystem can be active for more than one sample. The corresponding graph representation is illustrated in Figure 1.

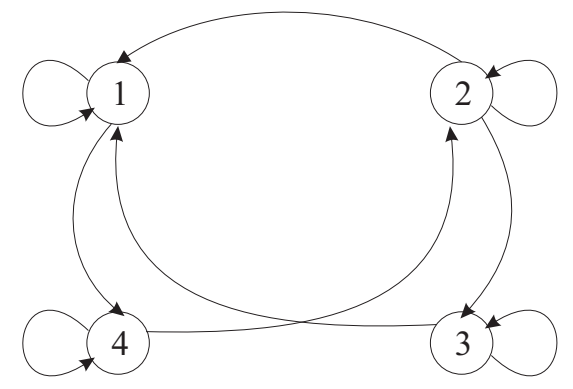

Fig. 1. Graph representation of the switching system in Example 1.

The graph is $\mathcal{G}=\{\mathcal{V}, \mathcal{E}\}$, with $\mathcal{V}=\{1,2,3,4\}$ and

$$
\begin{array}{r}
\mathcal{E}=\{(1,1),(1,4),(2,1),(2,2),(2,3),(3,1), \\
(3,3),(4,2),(4,4)\}
\end{array}
$$

A path $\mathcal{P}(1,3)$ is given by $\mathcal{P}(1,3)=[1,4,2,3]$. The length of the path $\mathcal{P}(1,3)=[1,4,2,3]$ is 3 .

Once activated, a subsystem may be active continuously for at least $p_{i}^{m} \in \mathbb{N}^{+}$and at most $p_{i}^{M} \in \mathbb{N}^{+}$samples, that are assumed known. Our goal is to design a switching observer such that the estimation error dynamics converge to zero, with any admissible switching law.

0 and $I$ denote the zero and identity matrices of appropriate dimensions, and a $(*)$ denotes the term induced by symmetry. The subscript $z+m$ (as in $A_{1, z+m}$ ) stands for the scheduling vector being evaluated at the current sample plus $m$ th instant, i.e., $\boldsymbol{z}(k+m)$.

In what follows, we will make use of the following results:

Lemma 1. [23] Consider a vector $\boldsymbol{x} \in \mathbb{R}^{n_{x}}$ and two matrices $Q=Q^{T} \in \mathbb{R}^{n_{x} \times n_{x}}$ and $R \in \mathbb{R}^{m \times n_{x}}$ such that $\operatorname{rank}(R)<n_{x}$. The two following expressions are equivalent:

1) $\boldsymbol{x}^{T} Q \boldsymbol{x}<0, \boldsymbol{x} \in\left\{\boldsymbol{x} \in \mathbb{R}^{n_{x}}, \boldsymbol{x} \neq 0, R \boldsymbol{x}=0\right\}$

2) $\exists M \in \mathbb{R}^{n_{x} \times m}$ such that $Q+M R+R^{T} M^{T}<0$

Analysis and design for TS models often lead to double-sum negativity problems of the form

$$
\boldsymbol{x}^{T} \sum_{i=1}^{r} \sum_{j=1}^{r} h_{i}\left(\boldsymbol{z}_{k}\right) h_{j}\left(\boldsymbol{z}_{k}\right) \Gamma_{i j} \boldsymbol{x}<0
$$

where $\Gamma_{i j}, i, j=1,2, \ldots, r$ are matrices of appropriate dimensions.

Lemma 2. [24] The double-sum (2) is negative, if

$$
\begin{aligned}
& \Gamma_{i i}<0 \\
& \Gamma_{i j}+\Gamma_{j i}<0, \quad i, j=1,2, \ldots, r, i<j
\end{aligned}
$$

Lemma 3. [25] The double-sum (2) is negative, if

$$
\begin{aligned}
& \Gamma_{i i}<0 \\
& \frac{2}{r-1} \Gamma_{i i}+\Gamma_{i j}+\Gamma_{j i}<0, \quad i, j=1,2, \ldots, r, i \neq j
\end{aligned}
$$

\section{OBSERVER DESIGN}

Lets us now consider observer design for the switching system (1), repeated here for convenience:

$$
\begin{aligned}
\boldsymbol{x}_{k+1} & =A_{j, z} \boldsymbol{x}_{k}+B_{j, z} \boldsymbol{u}_{k} \\
\boldsymbol{y}_{k} & =C_{j, z} \boldsymbol{x}_{k}
\end{aligned}
$$

We propose to use a switching observer of the form

$$
\begin{aligned}
\widehat{\boldsymbol{x}}_{k+1} & =A_{j, z} \widehat{\boldsymbol{x}}_{k}+B_{j, z} \boldsymbol{u}_{k}+H_{j, z}^{-1} L_{j, z}\left(\boldsymbol{y}_{k}-\widehat{\boldsymbol{y}}_{k}\right) \\
\widehat{\boldsymbol{y}}_{k} & =C_{j, z} \widehat{\boldsymbol{x}}_{k}
\end{aligned}
$$

for the $j$-th subsystem, the observer switching together with the observed subsystem. The matrices $H_{j, i}$ and $L_{j, i}, j=$ $1,2 \ldots, n_{\mathrm{s}}, i=1,2, \ldots, r$ are to be determined.

The error dynamics $\boldsymbol{e}_{k}=\boldsymbol{x}_{k}-\widehat{\boldsymbol{x}}_{k}$ using this observer, under the assumption that the scheduling variables are available online at sample $k$, can be written as

$$
\begin{aligned}
\boldsymbol{e}_{k+1} & =A_{j, z} \boldsymbol{e}_{k}-H_{j, z}^{-1} L_{j, z}\left(\boldsymbol{y}_{k}-\widehat{\boldsymbol{y}}_{k}\right) \\
& =\left(A_{j, z}-H_{j, z}^{-1} L_{j, z} C_{j, z}\right) \boldsymbol{e}_{k}
\end{aligned}
$$

which in itself is a switching system.

To derive the observer design conditions, meaning that the error dynamics (4) should be asymptotically stable, consider the switching Lyapunov function

$$
V\left(\boldsymbol{e}_{k}\right)=\boldsymbol{e}_{k}^{T} P_{m, j, z} \boldsymbol{e}_{k}
$$

defined during the switches, i.e., on the edges of the associated graph $\mathcal{G}=\{\mathcal{V}, \mathcal{E}\}$, with $\left(v_{m}, v_{j}\right) \in \mathcal{E}$.

Remark: If a subsystem $j$ may be active for several number of samples, the edge $\left(v_{j}, v_{j}\right)$ is also considered.

With this, the following result can be formulated:

Theorem 1. The error dynamics (4) is asymptotically stable, if there exist $P_{m, j, k}=P_{m, j, k}^{T}>0, H_{j, k},\left(v_{m}, v_{j}\right) \in \mathcal{E},\left(v_{j}, v_{l}\right) \in$ $\mathcal{E}, k=1,2, \ldots, r$, such that

$$
\left(\begin{array}{cc}
-P_{m, j, z} & (*) \\
H_{j, z} A_{j, z}-L_{j, z} C_{j, z} & -H_{j, z}-H_{j, z}^{T}+P_{j, l, z+1}
\end{array}\right)<0
$$

for all admissible paths $\mathcal{P}\left(v_{m}, v_{l}\right)=\left[v_{m}, v_{j}, v_{l}\right], v_{m} \in \mathcal{V}$. 
Proof. Consider the switching Lyapunov function (5), defined on the edges of the associated graph, with $\boldsymbol{e}_{k}^{T} P_{m, j, z} \boldsymbol{e}_{k}$ being active during the transition from vertex $m$ to vertex $j$. The difference in the Lyapunov function for two consecutive samples is

$$
\begin{aligned}
\Delta V & =\boldsymbol{e}_{k+1}^{T} P_{j, l, z+1} \boldsymbol{e}_{k+1}-\boldsymbol{e}_{k}^{T} P_{m, j, z} \boldsymbol{e}_{k} \\
& =\left(\begin{array}{c}
\boldsymbol{e}_{k} \\
\boldsymbol{e}_{k+1}
\end{array}\right)^{T}\left(\begin{array}{cc}
-P_{m, j, z} & 0 \\
0 & P_{j, l, z+1}
\end{array}\right)\left(\begin{array}{c}
\boldsymbol{e}_{k} \\
\boldsymbol{e}_{k+1}
\end{array}\right)
\end{aligned}
$$

where $\left[v_{m}, v_{j}, v_{l}\right]$ is an admissible path.

During the transition for $j$ to $l$, the dynamics of the error system are described by

$$
\left(\begin{array}{ll}
A_{j, z}-H_{j, z}^{-1} L_{j, z} C_{j, z} & -I
\end{array}\right)\left(\begin{array}{c}
\boldsymbol{e}_{k} \\
\boldsymbol{e}_{k+1}
\end{array}\right)=0
$$

Using Lemma 1, the difference in the Lyapunov function is negative, if there exists $M$ such that

$$
\begin{aligned}
& \left(\begin{array}{cc}
-P_{m, j, z} & 0 \\
0 & P_{j, l, z+1}
\end{array}\right) \\
& +M\left(A_{j, z}-H_{j, z}^{-1} L_{j, z} C_{j, z} \quad-I\right)+(*)<0
\end{aligned}
$$

By choosing

$$
M=\left(\begin{array}{c}
0 \\
H_{j, z}
\end{array}\right)
$$

we have directly (6).

Using Lemma 3 LMI conditions can be formulated, as follows:

Corollary 1. The error dynamics (4) is asymptotically stable, if there exist $P_{m, j, k}=P_{m, j, k}^{T}>0, H_{j, k},\left(v_{m}, v_{j}\right) \in \mathcal{E}, k, l=$ $1,2, \ldots, r$, such that

$$
\begin{aligned}
& \Gamma_{k k}^{m, j, l, \gamma}<0 \\
& \frac{2}{r-1} \Gamma_{k k}^{m, j, l, \gamma}+\Gamma_{k \beta}^{i, j, l, \gamma}+\Gamma_{\beta k}^{i, j, l, \gamma}<0 \\
& k, \beta, \gamma=1,2, \ldots, r
\end{aligned}
$$

with

$$
\Gamma_{k \beta}^{m, j, l, \gamma}=\left(\begin{array}{cc}
-P_{m, j, k} & (*) \\
H_{j, k} A_{j, \beta}-L_{j, k} C_{j, \beta} & -H_{j, k}+(*)+P_{j, l, \gamma}
\end{array}\right)
$$

for all admissible paths $\mathcal{P}\left(v_{m}, v_{l}\right)=\left[v_{m}, v_{j}, v_{l}\right], v_{j} \in \mathcal{V}$.

Remark: In order to reduce the conservativeness by exploiting the knowledge available of the switching sequence, one can also use the observer

$$
\begin{aligned}
\widehat{\boldsymbol{x}}_{k+1} & =A_{j, z} \widehat{\boldsymbol{x}}_{k}+B_{j, z} \boldsymbol{u}_{k}+H_{m, j, z}^{-1} L_{m, j, z}\left(\boldsymbol{y}_{k}-\widehat{\boldsymbol{y}}_{k}\right) \\
\widehat{\boldsymbol{y}}_{k} & =C_{j, z} \widehat{\boldsymbol{x}}_{k}
\end{aligned}
$$

for the $j$-th subsystem, if the last switch has been from vertex $m$ to vertex $j$.

The error dynamics $\boldsymbol{e}_{k}=\boldsymbol{x}_{k}-\widehat{\boldsymbol{x}}_{k}$ using this observer, can be written as

$$
\begin{aligned}
\boldsymbol{e}_{k+1} & =A_{j, z} \boldsymbol{e}_{k}-H_{m, j, z}^{-1} L_{m, j, z}\left(\boldsymbol{y}_{k}-\widehat{\boldsymbol{y}}_{k}\right) \\
& =\left(A_{j, z}-H_{m, j, z}^{-1} L_{m, j, z} C_{j, z}\right) \boldsymbol{e}_{k}
\end{aligned}
$$

Following the same steps as above, and in Finsler's lemma choosing

$$
M=\left(\begin{array}{c}
0 \\
H_{m, j, z}
\end{array}\right)
$$

we have

Corollary 2. The error dynamics (8) is asymptotically stable, if there exist $P_{m, j, k}=P_{m, j, k}^{T}>0, H_{m, j, k},\left(v_{m}, v_{j}\right) \in \mathcal{E}$, $\left(v_{j}, v_{l}\right) \in \mathcal{E}, k=1,2, \ldots, r$, such that

$$
\left(\begin{array}{cc}
-P_{m, j, z} & (*) \\
H_{m, j, z} A_{j, z}-L_{m, j, z} C_{j, z} & -H_{m, j, z}+(*)+P_{j, l, z+1}
\end{array}\right)<0
$$

for all admissible paths $\mathcal{P}\left(v_{m}, v_{l}\right)=\left[v_{m}, v_{j}, v_{l}\right], v_{m} \in \mathcal{V}$.

The result above can be further extended to take into account more previous switches. However, this comes with the added computational cost and will eventually lead to considering all possible switching trajectories. To avoid this, but still reduce the conservativeness of the approach, let us now consider the $\alpha$-sample variation of the Lyapunov function. As proven by [26], a system is asymptotically stable, if the associated Lyapunov function decreases every $\alpha$ samples, $\alpha \geq 1$, instead of every sample. Thus, for observer design, let us consider the error dynamics (4) and the Lyapunov function (5). Then, the following result can be formulated:

Theorem 2. The error dynamics (4) is asymptotically stable, if there exist $\alpha \in \mathbb{N}^{+}, P_{i, j, k}=P_{i, j, k}^{T}>0, H_{j, k},\left(v_{i}, v_{j}\right) \in \mathcal{E}$, $k=1,2, \ldots, r$, such that

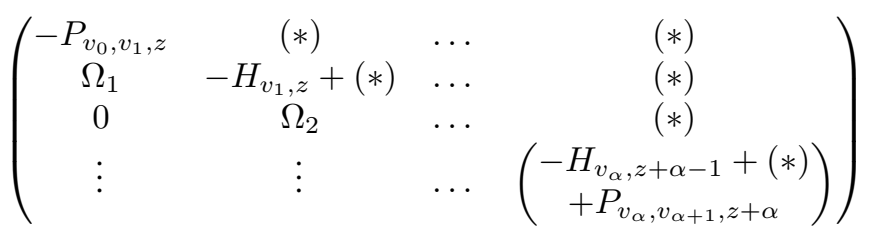

for all admissible paths $\mathcal{P}\left(v_{0}, v_{\alpha+1}\right)=\left[v_{0}, v_{1}, \ldots, v_{\alpha+1}\right]$, where $\Omega_{i}=H_{v_{i}, z+i-1} A_{v_{i}, z+i-1}-L_{v_{i}, z+i-1} C_{v_{i}, z+i-1}$.

Proof. Consider the switching Lyapunov function (5), defined on the edges of the associated graph, with $P_{v_{i}, v_{j}, z}$ being active during the transition from vertex $i$ to vertex $j$. The difference in the Lyapunov function for $\alpha$ consecutive samples is

$$
\begin{aligned}
\Delta V= & \boldsymbol{e}_{k+\alpha}^{T} P_{v_{\alpha}, v_{\alpha+1}, z+\alpha} \boldsymbol{e}_{k+\alpha}-\boldsymbol{e}_{k}^{T} P_{v_{0}, v_{1}, z} \boldsymbol{e}_{k} \\
= & \left(\begin{array}{c}
\boldsymbol{e}_{k} \\
\boldsymbol{e}_{k+\alpha}
\end{array}\right)^{T}\left(\begin{array}{cc}
-P_{v_{0}, v_{1}, z} & 0 \\
0 & P_{v_{\alpha}, v_{\alpha+1}, z+\alpha}
\end{array}\right) \\
& \cdot\left(\begin{array}{c}
\boldsymbol{e}_{k} \\
\boldsymbol{e}_{k+\alpha}
\end{array}\right)
\end{aligned}
$$

where $\left[v_{0}, v_{1}, \ldots, v_{\alpha+1}\right]$ is an admissible path.

Along the switching sequence $\left[v_{0}, v_{1}, \ldots, v_{\alpha+1}\right]$, the error 
dynamics are described by

$$
\left(\begin{array}{ccccc}
G_{1} & -I & 0 & \ldots & 0 \\
0 & G_{2} & -I & \ldots & 0 \\
\vdots & \vdots & & \vdots & \vdots \\
0 & 0 & 0 & \ldots & -I
\end{array}\right)\left(\begin{array}{c}
e_{k} \\
e_{k+1} \\
\vdots \\
e_{k+\alpha}
\end{array}\right)=0
$$

with $G_{i}=A_{v_{i}, z+i-1}-H_{v_{i}, z+i-1}^{-1} L_{v_{i}, z+i-1} C_{v_{i}, z+i-1}$.

Following the same steps as in the proof of Theorem 1, using Lemma 1, the difference is the Lyapunov function is negative, if there exists $M$ such that

$$
\begin{gathered}
\left(\begin{array}{ccccc}
-P_{v_{0}, v_{1}, z} & 0 & \ldots & & 0 \\
0 & 0 & \ldots & & 0 \\
\vdots & \vdots & \ldots & & \\
0 & 0 & \ldots & P_{v_{\alpha}, v_{\alpha+1}, z+\alpha}
\end{array}\right) \\
+M\left(\begin{array}{ccccc}
G_{1} & -I & 0 & \ldots & 0 \\
0 & G_{2} & -I & \ldots & 0 \\
\vdots & \vdots & & \vdots & \vdots \\
0 & 0 & 0 & \ldots & -I
\end{array}\right)+(*)<0
\end{gathered}
$$

with $G_{i}$ defined as above.

By choosing

$$
M=\left(\begin{array}{cccc}
0 & 0 & \ldots & 0 \\
H_{v_{1}, z} & 0 & \ldots & 0 \\
0 & H_{v_{2}, z+1} & \ldots & 0 \\
\vdots & \vdots & \ldots & \vdots \\
0 & 0 & \ldots & H_{v_{\alpha}, z+\alpha-1}
\end{array}\right)
$$

we have directly (10).

\section{EXAMPLE AND DISCUSSION}

Let us first illustrate the conditions of Theorem 1 on an example.

Example 2. Consider the switching system - actually a periodic switching system - illustrated in Figure 2. Assuming

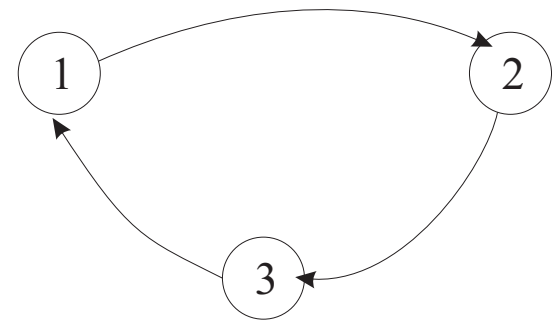

Fig. 2. Periodic switching system for Example 2.

that none of the subsystems may be active for more than one sample, i.e., $p^{m}=p^{M}=1$, the graph is $\mathcal{G}=\{\mathcal{V}, \mathcal{E}\}$, with $\mathcal{V}=\{1,2,3\}$ and

$$
\mathcal{E}=\{(1,2),(2,3),(3,1)\}
$$

. Consider the following local models of the TS system above:

$$
\begin{array}{lll}
A_{1,1}=\left(\begin{array}{ll}
0.61 & 0.10 \\
1.82 & 0.50
\end{array}\right) & A_{1,2}=\left(\begin{array}{ll}
0.21 & 1.59 \\
0.34 & 0.77
\end{array}\right) \\
C_{1,1}=\left(\begin{array}{ll}
1 & 0
\end{array}\right) & & C_{1,2}=\left(\begin{array}{ll}
1 & 0
\end{array}\right) \\
A_{2,1}=A_{2,2}=\left(\begin{array}{cc}
1.1 & 0 \\
0.2 & 0.8
\end{array}\right) & \\
C_{2,1}=\left(\begin{array}{ll}
0 & 0
\end{array}\right) & & C_{2,2}=\left(\begin{array}{ll}
0 & 0
\end{array}\right) \\
A_{3,1}=\left(\begin{array}{ll}
0.11 & 0.09 \\
0.09 & 0.07
\end{array}\right) & A_{3,2}=\left(\begin{array}{ll}
0.32 & 0.30 \\
0.09 & 0.38
\end{array}\right) \\
C_{3,1}=\left(\begin{array}{ll}
0 & 1
\end{array}\right) & C_{3,2}=\left(\begin{array}{ll}
0 & 0
\end{array}\right)
\end{array}
$$

The second subsystem is linear, but it is unstable and unobservable. The second local model of the third subsystem is again unstable and unobservable. Due to this, methods available in the literature yield unfeasible LMIs. However, using Lemma 2 to formulate LMI conditions for Theorem 1, we obtain ${ }^{1}$

$$
\begin{aligned}
& P_{1,2,1}=\left(\begin{array}{cc}
4.69 & -3.97 \\
-3.97 & 7.79
\end{array}\right) \quad P_{1,2,2}=\left(\begin{array}{cc}
4.69 & -3.97 \\
-3.97 & 7.79
\end{array}\right) \\
& P_{2,3,1}=\left(\begin{array}{cc}
3.45 & -3.35 \\
-3.35 & 5.05
\end{array}\right) \quad P_{2,3,2}=\left(\begin{array}{cc}
4.09 & -3.91 \\
-3.91 & 6.41
\end{array}\right) \\
& P_{3,1,1}=\left(\begin{array}{cc}
3.48 & -3.97 \\
-3.97 & 5.18
\end{array}\right) \quad P_{3,1,2}=\left(\begin{array}{cc}
4.74 & -5.67 \\
-5.67 & 7.39
\end{array}\right) \\
& H_{1,1}=\left(\begin{array}{cc}
4.83 & -2.45 \\
-2.50 & 6.26
\end{array}\right) \quad H_{1,2}=\left(\begin{array}{cc}
3.77 & -3.21 \\
-1.84 & 6.46
\end{array}\right) \\
& H_{2,1}=\left(\begin{array}{cc}
3.57 & -3.21 \\
-2.54 & 5.35
\end{array}\right) \quad H_{2,2}=\left(\begin{array}{cc}
3.57 & -3.21 \\
-2.54 & 5.35
\end{array}\right) \\
& H_{3,1}=\left(\begin{array}{cc}
4.55 & -5.05 \\
0.45 & 5.84
\end{array}\right) \quad H_{3,2}=\left(\begin{array}{cc}
4.14 & -4.61 \\
-4.85 & 6.27
\end{array}\right) \\
& L_{1,1}=\left(\begin{array}{c}
-2.14 \\
12.08
\end{array}\right) \quad L_{1,2}=\left(\begin{array}{l}
2.40 \\
3.45
\end{array}\right) \\
& L_{3,1}=\left(\begin{array}{l}
2.42 \\
4.27
\end{array}\right) \quad L_{3,2}=\left(\begin{array}{l}
3.89 \\
7.93
\end{array}\right)
\end{aligned}
$$

Note that there are no observer gains $L_{2,1}$ and $L_{2,2}$. This is because the second subsystem on its own is not observable. However, this observer is able to estimate the states of the switching system above. A trajectory of the error dynamics for the case when $\boldsymbol{x}(0)=[-1,1]^{T}$ and $\widehat{\boldsymbol{x}}(0)=0$ is illustrated in Figure 3. For the simulation, the membership functions used were $h_{1}=\frac{1}{2}\left(1-\sin x_{1}\right), h_{2}=1-h_{1}$, and for $L_{2,1}$ and $L_{2,2}$ zero matrices were used. The initial subsystem was the first one, from which the system switched according to the periodic law.

Let us now discuss the conditions developed for the $\alpha$ sample variation of the Lyapunov function. In the example above, the conditions required that the Lyapunov function decreases with every switch/every sample, i.e., for all $\left(v_{i}, v_{j}\right) \in$

\footnotetext{
${ }^{1}$ The values have been truncated to two decimal places.
} 


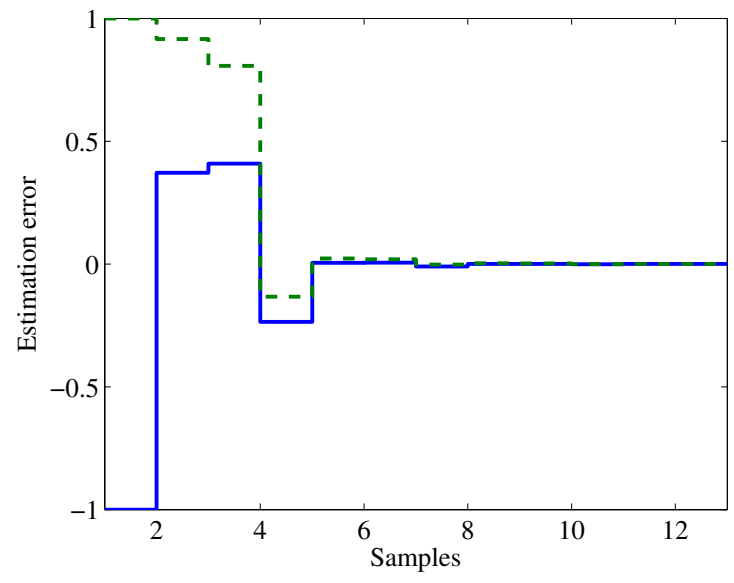

Fig. 3. Estimation error for Example 2.

$\mathcal{E}$. That is, we had the conditions:

$$
\begin{gathered}
\left(\begin{array}{cc}
-P_{1,2, z} & (*) \\
H_{2, z} A_{2, z}-L_{2, z} C_{2, z} & -H_{2, z}+(*)+P_{2,3, z+1}
\end{array}\right)<0 \\
\left(\begin{array}{cc}
-P_{2,3, z} & (*) \\
H_{3, z} A_{3, z}-L_{3, z} C_{3, z} & -H_{3, z}+(*)+P_{3,1, z+1}
\end{array}\right)<0 \\
\left(\begin{array}{cc}
-P_{3,1, z} & (*) \\
H_{1, z} A_{1, z}-L_{1, z} C_{1, z} & -H_{1, z}+(*)+P_{1,2, z+1}
\end{array}\right)<0
\end{gathered}
$$

On the other hand, a 2-sample variation means that the Lyapunov function has to decrease along paths of length 2, i.e., we have the conditions:

$$
\begin{aligned}
& \left(\begin{array}{ccc}
-P_{1,2, z} & (*) & (*) \\
\left(\begin{array}{c}
H_{2, z} A_{2, z} \\
-L_{2, z} C_{2, z}
\end{array}\right) & -H_{2, z}+(*) & (*) \\
0 & \left(\begin{array}{c}
H_{3, z+1} A_{3, z+1} \\
-L_{3, z+1} C_{3, z+1}
\end{array}\right) & \left(\begin{array}{c}
-H_{3, z+1}+(*) \\
+P_{3,1, z+2}
\end{array}\right)
\end{array}\right)<0 \\
& \left(\begin{array}{c}
-P_{2,3, z} \\
H_{3, z} A_{3, z} \\
-L_{3, z} C_{3, z}
\end{array}\right) \\
& \text { (*) } \\
& \left(\begin{array}{c}
-P_{3,1, z} \\
H_{1, z} A_{1, z} \\
-L_{1, z} C_{1, z}
\end{array}\right) \\
& \begin{array}{c}
-H_{3, z}+(*) \\
\left(\begin{array}{c}
H_{1, z+1} A_{1, z+1} \\
-L_{1, z+1} C_{1, z+1}
\end{array}\right)
\end{array} \\
& \left.\begin{array}{c}
-H_{1, z}+(*) \\
H_{2, z+1} A_{2, z+1} \\
-L_{2, z+1} C_{2, z+1}
\end{array}\right) \\
& \left.\left(\begin{array}{c}
-H_{2, z+1}+(*) \\
+P_{2,3, z+2}
\end{array}\right)\right)^{<}
\end{aligned}
$$

Furthermore, a 3-sample variation means that the Lyapunov function has to decrease along paths of length 3 , which, in this case, is equivalent to the whole period of switching.

In the example above, one of the reasons for which the LMIs are feasible is that the subsystems may be active for a finite number of samples. In the case considered above, the switching occurs at every sample, i.e., each subsystem is active for one sample. Feasible LMIs are also obtained if the subsystems may be active for at most 2 samples. However, assuming that each subsystem, once activated may remain active for an infinite number of samples, the corresponding LMIs become unfeasible.
We assumed that the switching sequence is not known in advance and it cannot be directly influenced. Due to this assumption the conditions require that the estimation error dynamics is stable if the Lyapunov function decreases along every path of length $\alpha$. This is the worst-case assumption, i.e., all possible combinations on switches between the estimation error subsystems have to be taken into account. If the switching sequence can be chosen, or it is known in advance, the conditions can be relaxed.

A shortcoming of the proposed conditions is the computational complexity of generating all the switching paths of length $\alpha$, in particular for large $\alpha$ and large-scale switching systems and in consequence, a large number of LMIs that has to be solved. However, reducing the conservativeness of the conditions by introducing additional sums in the Lyapunov function increases the number of LMIs.

\section{Conclusions}

In this paper we developed observer design conditions for discrete-time switching systems represented by Takagi-Sugeno fuzzy models, by using a switching Lyapunov function defined on the switches. We assumed that the switching sequence is not known in advance and it cannot be directly influenced. The developed conditions have been formulated as LMIs.

\section{ACKNOWLEDGMENT}

This work was supported by a grant of the Romanian National Authority for Scientific Research, CNCS - UEFISCDI, project number PN-II-RU-TE-2011-3-0043, contract number 74/05.10.2011, by International Campus on Safety and Intermodality in Transportation the European Community, the Délegation Régionale à la Recherche et à la Technologie, the Ministére de L'Enseignement supérieur et de la Recherche the Region Nord Pas de Calais and the Centre Nationale de la Recherche Scientifique: the authors gratefully acknowledge the support of these institutions.

\section{REFERENCES}

[1] J. Daafouz, P. Riedinger, and C. Iung, "Stability analysis and control synthesis for switched systems: a switched Lyapunov function approach," IEEE Transactions on Automatic Control, vol. 47, no. 11, pp. 18831887, 2002.

[2] T. Takagi and M. Sugeno, "Fuzzy identification of systems and its applications to modeling and control," IEEE Transactions on Systems, Man, and Cybernetics, vol. 15, no. 1, pp. 116-132, 1985.

[3] K. Tanaka, T. Ikeda, and H. Wang, "Fuzzy regulators and fuzzy observers: relaxed stability conditions and LMI-based designs," IEEE Transactions on Fuzzy Systems, vol. 6, no. 2, pp. 250-265, 1998.

[4] K. Tanaka and H. O. Wang, Fuzzy Control System Design and Analysis: A Linear Matrix Inequality Approach. New York, NY, USA: John Wiley \& Sons, 2001.

[5] A. Sala, T. M. Guerra, and R. Babuška, "Perspectives of fuzzy systems and control," Fuzzy Sets and Systems, vol. 156, no. 3, pp. 432-444, 2005.

[6] T. M. Guerra and L. Vermeiren, "LMI-based relaxed nonquadratic stabilization conditions for nonlinear systems in the Takagi-Sugeno's form," Automatica, vol. 40, no. 5, pp. 823-829, 2004.

[7] A. Kruszewski, R. Wang, and T. M. Guerra, "Nonquadratic stabilization conditions for a class of uncertain nonlinear discrete time TS fuzzy models: A new approach," IEEE Transactions on Automatic Control, vol. 53, no. 2, pp. 606-611, 2008.

[8] L. A. Mozelli, R. M. Palhares, F. O. Souza, and E. M. A. M. Mendes, "Reducing conservativeness in recent stability conditions of TS fuzzy systems," Automatica, vol. 45, no. 6, pp. 1580-1583, 2009. 
[9] B. Ding, H. Sun, and P. Yang, "Further studies on LMI-based relaxed stabilization conditions for nonlinear systems in Takagi-Sugeno's form," Automatica, vol. 42, no. 3, pp. 503-508, 2006.

[10] J. Dong and G. Yang, "Dynamic output feedback $H_{\infty}$ control synthesis for discrete-time T-S fuzzy systems via switching fuzzy controllers," Fuzzy Sets and Systems, vol. 160, no. 19, pp. 482-499, 2009.

[11] D. H. Lee, J. B. Park, and Y. H. Joo, "Approaches to extended nonquadratic stability and stabilization conditions for discrete-time TakagiSugeno fuzzy systems," Automatica, vol. 47, no. 3, pp. 534-538, 2011.

[12] K. Tanaka, M. Iwasaki, and H. O. Wang, "Switching control of an R/C hovercraft: stabilization and smooth switching," IEEE Transactions on Systems, Man and Cybernetics, Part B, vol. 31, no. 6, pp. 853-863, 2001.

[13] H. K. Lam, F. H. F. Leung, and P. K. S. Tam, "A switching controller for uncertain nonlinear systems," IEEE Control Systems Magazine, vol. 22, no. 1, pp. 1-14, 2002.

[14] H. K. Lam, F. H. F. Leung, and Y. S. Lee, "Design of a switching controller for nonlinear systems with unknown parameters based on a fuzzy logic approach," IEEE Transactions on Systems, Man and Cybernetics, Part B, vol. 34, no. 2, pp. 1068-1074, 2004.

[15] H. Ohtake, K. Tanaka, and H. O. Wang, "Switching fuzzy controller design based on switching Lyapunov function for a class of nonlinear systems," IEEE Transactions on Systems, Man and Cybernetics, Part B, vol. 36, no. 1, pp. 13-23, 2006.

[16] G. Feng, "Controller synthesis of fuzzy dynamic systems based on piecewise Lyapunov functions," IEEE Transactions on Fuzzy Systems, vol. 11 , no. 5 , pp. 605-612, 2003.

[17],$- ~ " H_{\infty}$ controller design of fuzzy dynamic systems based on piecewise Lyapunov functions," IEEE Transactions on Systems, Man, and Cybernetics, Part B: Cybernetics, vol. 34, no. 1, pp. 283-292, 2004.

[18] J. C. Doo, S. L. Seung, and P. PooGyeon, "Output-feedback control of discrete-time switching fuzzy system," in Proceedings of the IEEE International Conference on Fuzzy Systems, St. Luis, MO, USA, May 2003, pp. 441-446.

[19] J. Dong and G. Yang, " $H_{\infty}$ controller synthesis via switched PDC scheme for discrete-time T-S fuzzy systems," IEEE Transactions on Fuzzy Systems, vol. 17, no. 3, pp. 544-555, 2009.

[20] M. Jungers, E. B. Castelan, S. Tarbouriech, and J. Daafouz, "Finite $L_{2}$-induced gain and $\lambda$-contractivity of discrete-time switching systems including modal nonlinearities and actuator saturations," Nonlinear Analysis: Hybrid Systems, vol. 5, pp. 289-300, 2011.

[21] M. Dehghan and C.-J. Ong, "Computations of mode-dependent dwell times for discrete-time switching system," Automatica, vol. 49, pp. 1804-1808, 2013.

[22] E. De Santis, "On location observability notions for switching systems," Systems \& Control Letters, vol. 60, pp. 807-814, 2011.

[23] R. E. Skelton, T. Iwasaki, and K. Grigoriadis, A Unified Approach to Linear Control Design. Taylor \& Francis, 1998.

[24] H. Wang, K. Tanaka, and M. Griffin, "An approach to fuzzy control of nonlinear systems: stability and design issues," IEEE Transactions on Fuzzy Systems, vol. 4, no. 1, pp. 14-23, 1996.

[25] H. Tuan, P. Apkarian, T. Narikiyo, and Y. Yamamoto, "Parameterized linear matrix inequality techniques in fuzzy control system design," IEEE Transactions on Fuzzy Systems, vol. 9, no. 2, pp. 324-332, 2001.

[26] A. Kruszewski and T. M. Guerra, "Stabilization of a class of nonlinear model with periodic parameters in the Takagi-Sugeno form," in Proceedings of the IFAC Workshop Periodic Control Systems, Saint Petersburg, Russia, 2007, pp. 1-6. 